\title{
Are retrograde resonances possible in multi-planet systems?^
}

\author{
J. Gayon and E. Bois
}

\author{
Nice Sophia-Antipolis University, CNRS, Observatoire de la Côte d'Azur, Laboratoire Cassiopée, BP 4229, 06304 Nice Cedex 4 , \\ France \\ e-mail: Julie.Gayon@oca.eu.fr; Eric.Bois@oca.eu
}

Received 10 August 2007 / Accepted 7 January 2008

\begin{abstract}
Context. Most multi-planetary systems are characterized by hot-Jupiters close to their central star, moving on eccentric orbits. From a dynamical point of view, compact multi-planetary systems form a specific class of the general $N$-body problem (where $N \geq 3$ ). Moreover, extrasolar planets are found in prograde orbits about their host star, and often in mean motion resonances (MMR).

Aims. In a first step, we study theoretically a new stabilizing mechanism suitable for compact two-planet systems. This mechanism involves counter-revolving orbits forming a retrograde MMR. In a second step, we investigate the feasibility of planetary systems hosting counter-revolving planets. Dynamical stability, observations, and formation processes of these systems are analyzed and discussed.

Methods. To characterize the dynamical behavior of multi-dimensional planetary systems, we apply our technique of global dynamics analysis based on the MEGNO indicator (Mean Exponential Growth factor of Nearby Orbits) that provides the fine structure of the phase space. In a few cases of possible counter-revolving configurations, we carry out new fits to the observations using the Pikaia genetic algorithm. A statistical study of the stability in the neighborhood of different observed, planetary-systems is completed using a Monte-Carlo method.

Results. We analyse the observational data for the HD 73526 planetary system and find that counter-revolving configurations may be consistent with the observational data. We highlight the fine and characteristic structure of retrograde MMRs. We demonstrate that retrograde resonances open a family of stabilizing mechanisms involving new apsidal precession behaviors.

Conclusions. Considering two possible formation mechanisms (free-floating planet and Slingshot model), we conclude that counterrevolving configurations are feasible.
\end{abstract}

Key words. celestial mechanics - stars: planetary systems - methods: numerical

\section{Introduction}

At present, 271 extrasolar planets have been detected around 233 stars (both solar and non-solar type) ${ }^{1}$. Among them, there are 25 multiple-planet systems: 17 two-planet systems (e.g. HD 82943, 47 UMa, HD 108874, HD 128311), 6 three-planet systems (e.g. $v$ And, HD 69830, Gliese 876, Gliese 581), 1 fourplanet system (HD 160691) and more recently 1 five-planet system (55 CnC). Observations indicate that Mean Motion Resonances (MMR) frequently occur for planets of multipleplanet systems: Gliese 876 (e.g. Rivera et al. 2005), HD 82943 (e.g. Ji et al. 2003; Mayor et al. 2004) and HD 128311 (Vogt et al. 2005) are in 2:1 MMR, HD 202206 is in 5:1 MMR (Correia et al. 2005), while 47 UMa is close to a 7:3 (Fischer et al. 2002) or 8:3 commensurability (Fischer et al. 2003).

This work is devoted to compact multi-planetary systems, characterized by (a) giant Jupiter-like planets found close to their central star, and (b) high eccentricities. These two peculiarities lead to strong gravitational interactions between the planets and may result in an unstable, dynamical behavior. However, we observe many such planetary systems suggesting that they are stable, and raising the question of why they are stable. From a

\footnotetext{
* Movies are available in electronic form at

http: //www . aanda. org

1 January, the 11th, 2008, http://exoplanet.eu/catalog.php
}

dynamical point of view, compact multi-planetary systems form a specific class of the general $N$-body problem (with $N \geq 3$ ) whose analytical solutions are not necessarily known. A stability analysis of planetary systems, using numerical methods to explore multi-dimensional parameter space, typically leads to stability maps in which rare islands of stability can be identified amidst large chaotic zones. The underlying mechanisms for these stability zones must be identified.

In 2002, Kiseleva-Eggleton et al. (2002) showed that the currently-published, orbital parameters place the planetary systems HD 12661, HD 38529, HD 37124, and HD 160691 in very different situations from the point of view of dynamical distribution. Since this first study of the comparative stability of multiplanetary systems, many studies have been carried out in this direction. The role of the orbital mean motion resonances, in particular with a 2:1 ratio, has been intensively studied by several research groups (for example Hadjidemetriou 2002; Lee \& Peale 2002, 2003; Bois et al. 2003; Ji et al. 2003; Ferraz-Mello et al. 2005b; Psychoyos \& Hadjidemetriou 2005; Beaugé et al. 2006). As a result, it has been discovered that an extrasolar planetary system, even with large planetary masses and eccentricities, can be stable if planetary orbits are close to stable, resonant, and periodic orbits. It has also been established (see e.g. Chiang \& Murray 2002; Lee \& Peale 2002; Libert \& Henrard 2006) that orbits in a large number of compact multi-planet systems, 
Table 1. Orbital parameters of the HD 73526 and HD 160691 planetary systems. Data come from Tinney et al. (2006) and McCarthy et al. (2004) respectively.

\begin{tabular}{|c|c|c|c|c|c|c|c|}
\hline Planets & $\begin{array}{l}M_{\text {star }} \\
\left(M_{\odot}\right)\end{array}$ & $\begin{array}{c}m_{\mathrm{P}} \sin i_{l} \\
\left(M_{J}\right)\end{array}$ & $\begin{array}{c}P \\
\text { (days) }\end{array}$ & $\begin{array}{c}a \\
(\mathrm{AU})\end{array}$ & $e$ & $\begin{array}{c}\omega \\
(\mathrm{deg})\end{array}$ & $\begin{array}{c}M \\
(\mathrm{deg})\end{array}$ \\
\hline HD 73526b & & $2.9 \pm 0.2$ & $188.3 \pm 0.9$ & $0.66 \pm 0.01$ & $0.19 \pm 0.05$ & $203 \pm 9$ & $86 \pm 13$ \\
\hline HD $73526 c$ & to & $2.5 \pm 0.3$ & $377.8 \pm 2.4$ & $1.05 \pm 0.02$ & $0.14 \pm 0.09$ & $13 \pm 76$ & $82 \pm 27$ \\
\hline HD 160691b & & $1.67 \pm 0.11$ & $645.5 \pm 3$ & $1.50 \pm 0.02$ & $0.20 \pm 0.03$ & $294 \pm 9$ & 0 \\
\hline HD 160691c & $1.08=$ & $3.10 \pm 0.71$ & $2986 \pm 30$ & $4.17 \pm 0.07$ & $0.57 \pm 0.1$ & $161 \pm 8$ & $12.6 \pm 11.2$ \\
\hline
\end{tabular}

are locked in Apsidal Synchronous Precessions (ASP hereafter), i.e. that the apsidal lines precess, on average, at the same rate ${ }^{2}$. A solution involving both MMRs and ASP describes well the stability of eccentric, compact multi-planetary systems, but may not however be unique. We note, for example, that other multiplanetary systems have been found to be mainly controlled by secular dynamics (cf. Michtchenko et al. 2006; Libert \& Henrard 2006; Ji et al. 2007). In the present paper, we illustrate theoretically that other mechanisms can in addition provide the stability in multi-planetary systems.

In the case of the HD 73526 system (2:1 MMR), Tinney et al. (2006) found stability over 1 Myr. Based on the analytical classification of Hadjidemetriou (2002) established according to a hierarchy of masses and eccentricities, this system could instead be classified as unstable. Hadjidemetriou's classification may however be too general to disprove the stability found by Tinney et al. (2006). Be that as it may, we use the same data as Tinney et al. (see Table 1) and our numerical method is outlined in the following section. Exploring the stability of the HD 73526 system in orbital parameter space, we find large chaotic regions. We find that the published data can even be described by a chaotic behavior. We note however that Tinney et al. (2006) used a different definition of stability ${ }^{3}$. Of course, we cannot exclude that the observational data were insufficient to allow a reliable orbital fit or the fit itself was not adequate. On the other hand, it is also possible that the underlying assumption of two prograde orbits is wrong. When placing one of the two planets on a retrograde orbit (which forms a system with counter-revolving planets), the stability region becomes very large. We will show below that this does not imply that the orbital fit is consistent with this stability zone. It implies that, in the neighborhood of the observational point, we can theoretically find stable solutions for counter-revolving configurations. To distinguish between two resonance cases when both planets are in prograde orbits, or when one planet is on a retrograde orbit, we call them prograde and retrograde resonances, respectively.

Presently, all known extrasolar planets in multiple systems are believed to revolve in the same direction about their corresponding central star. Most fitted, orbital elements are derived

\footnotetext{
${ }^{2}$ For the study of 3D, full 3-body problems, we introduced the terminology ASP, for expressing that the apsidal lines precess on average in a 3D space at the same rate: see Bois (2005); Bois et al. (2005). We note that in the planar case this phenomenon is also called "apsidal corotation" (ACR; Beaugé et al. 2003). In a number of papers, one may also find the incorrect expression "apsidal secular resonances" (ASR). ACR and ASP are in general not true secular resonances, as highlighted by Ferraz-Mello et al. (2005a).

${ }^{3}$ In the paper of Tinney et al. (2006), the claim of stability is obtained from the dynamical behaviors of the resonant angles related to the 2:1 MMR, rather than by characterizations of quasi-periodicity of the orbital solution. Besides, the notion of stability is only presumed to be acquired by the simple absence of planet ejection. We use instead the usual definition of stability related to quasi-periodicity (see Sect. 2) and suitable for conservative dynamical systems.
}

by assuming prograde orbits. This is expected according to current theories for planetary formation in a circumstellar disk. In order to obtain a planet in retrograde resonance, an additional event is necessary such as violent, dynamical evolution of the planetary system, or a capture of the retrograde planet. In our Solar System, comets and the planetary satellites of Neptune, Saturn and Jupiter are known to have retrograde orbits. It is, therefore, important to investigate the stability of exoplanetary systems with a retrograde planet in particular if the observations do not yield a stable system when assuming all planets on prograde orbits.

In Sect. 2, we present our method of global dynamics analysis. We show that there exists theoretically initial conditions in the vicinity of observational data such that stability is only possible for a counter-revolving configuration. It raises the question of whether such a configuration is consistent with the observational data of a given system (Sect. 3). In Sect. 4, we focus on the statistical occurence of stable solutions related to both prograde and retrograde resonances. This statistical approach is applied to three systems in 2:1 MMR and two systems in 5:1 MMR. If such systems harboring counter-revolving planets exist, we must also consider how they form: we discuss this issue in Sect. 5. By analyzing the parameter space in the vicinity of the best-fit of the HD 73526 planetary system, we highlight the fine structure of the 2:1 retrograde resonance (Sects. 6 and 7), and the nature of associated apsidal precessions (Sect. 8). In addition, we complete an analoguous study for a theoretical system in 5:1 retrograde MMR (Sect. 9).

\section{Method}

In order to explore the stability in the parameter space of known exoplanetary system in the case of retrograde resonance, we use the MEGNO (Mean Exponential Growth factor of Nearby Orbits) method proposed by Cincotta \& Simò (2000). This method provides relevant information on the global dynamics of multi-dimensional, Hamiltonian systems and the fine structure of their phase space (Cincotta et al. 2002). It simultaneously yields a good estimate of the Lyapunov Characteristic Numbers (LCN) with a comparatively small computational effort (Cincotta \& Giordano 2000). It provides a clear picture of resonance structures, location of stable and unstable periodic orbits, as well as a measure of hyperbolicity in chaotic domains (i.e. the rate of divergence of unstable orbits). Using the MEGNO technique, we have built the MIPS (Megno Indicator for Planetary Systems) package specifically devoted to studying multi-dimensional planetary systems and their conditions of dynamical stability. We use the property of stability in the Poisson sense: stability is related to the preservation of a neighborhood related to the initial position of the trajectory. Moreover, in the Poincaré-Lyapunov sense applied to conservative systems, when quasi-periodic orbits remain confined within certain limits, they are called stable. We note that chaotic, in the Poincare sense 
(a)

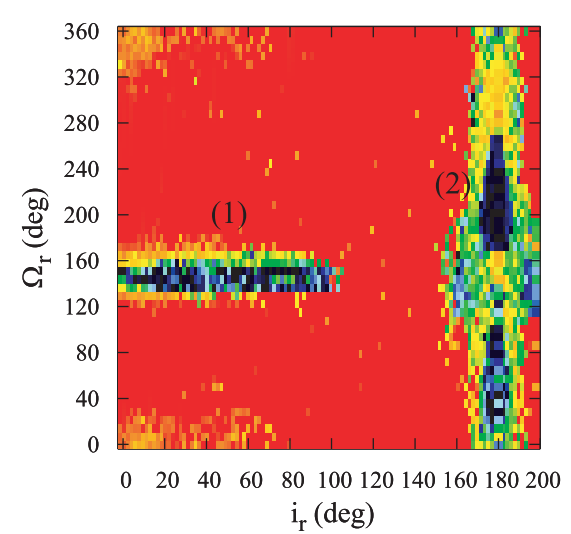

(b)

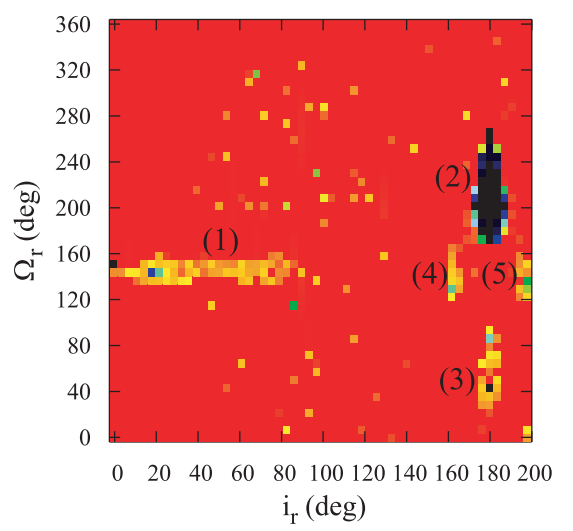

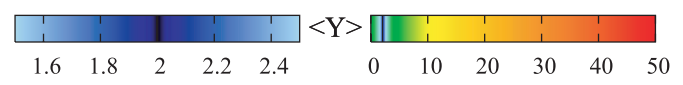

Fig. 1. Stability maps for the HD 73526 planetary system in the $\left[i_{\mathrm{r}}, \Omega_{\mathrm{r}}\right]$ parameter space. Panel a) is plotted with an integration time of 5000 yrs while planel b) is plotted over one order of magnitude longer, namely 50000 years. One observes the disappearance of the stable island (1). Only the island (2) survives for longest timescales. Initial conditions come from the best fit of Tinney et al. (2006) (see Table 1). Relative inclinations and relative longitudes of nodes are defined as follows: $i_{\mathrm{r}}=i_{\mathrm{c}}-i_{\mathrm{b}}$ and $\Omega_{\mathrm{r}}=\Omega_{\mathrm{c}}-\Omega_{\mathrm{b}}$ (by convention, at $t=0, \Omega_{\mathrm{b}}=0^{\circ}$ and $i_{\mathrm{b}}=0^{\circ}$ ). Resolution of the grid is $110 \times 50$ for panel a), $55 \times 50$ for panel b). Black and dark-blue colors represent highly stable orbits $(\langle Y\rangle=2 \pm 3 \%$ and $\langle Y\rangle=2 \pm 5 \%$ respectively, $\langle Y\rangle$ being the MEGNO indicator value) while warm colors refer to highly unstable orbits $(\langle Y\rangle \gg 2)$.

means that the dynamical behavior is not quasi-periodic (according to the conventional definition used for conservative dynamical systems) and does not necessarily mean that the system will disintegrate during limited period of time. We have already successfully applied the MEGNO technique with the MIPS package to the study of dynamical stability of extrasolar planetary systems in a series of previous papers (see e.g. Bois et al. 2003, 2004). In the MIPS package, let us note that the $i_{\mathrm{b}, \mathrm{c}}$ inclination parameters refer to the dynamical, orbital-element independent of the $\sin i_{l}$ line-of-sight inclination factor ${ }^{4}$.

By applying the observational data of the HD 73526 planetary system (see Table 1$)^{5}$, and scanning the non-determined elements, namely the $i_{\mathrm{r}}$ relative inclination $\left(i_{\mathrm{r}}=i_{\mathrm{c}}-i_{\mathrm{b}}\right)$ and the $\Omega_{\mathrm{r}}$ relative longitude of nodes $\left(\Omega_{\mathrm{r}}=\Omega_{\mathrm{c}}-\Omega_{\mathrm{b}}\right)$, we find two main islands of stability, as shown in Fig. 1a. The first (1) is obtained for $i_{\mathrm{r}} \in\left[8^{\circ}, 97^{\circ}\right]$, and the other (2) for very high relative inclinations, namely $i_{\mathrm{r}} \in\left[173^{\circ}, 187^{\circ}\right]$. In this stability map, we highlight that stability does not allow coplanar prograde orbits.

The purpose of fast-chaos indicators, and in particular of MEGNO, is to predict dynamical behavior over a long timescale using short integration times. Our integration times do not mean stability times or prediction limits but, using the MEGNO indicator, they express the minimal times for knowing trajectory future. As a result of the principle of MEGNO, stability is generally acquired for timescales far longer than the integration times. The ratio of "prediction time" to "integration time" achieved by MEGNO, is optimal.

The MIPS maps presented in this paper were confirmed by a second global analysis technique (e.g. Marzari et al. 2006),

\footnotetext{
${ }^{4}$ In the present paper, masses of planets remain untouched whatever the mutual inclinations may be. Our reference frame is related to the planetary system itself, then dynamically autonomous relative to observations. Moreover, scanning the phase space, our stability maps express the variations of two explicit parameters, without implicit and external relations.

${ }^{5}$ with in addition at $t=0, \Omega_{\mathrm{b}}=\Omega_{\mathrm{c}}=0, i_{\mathrm{b}}=0$, and $i_{\mathrm{c}}=1^{\circ}$ (because of gravitational interactions of the whole 3-body problem, the relative inclination $i_{\mathrm{r}}=i_{\mathrm{c}}-i_{\mathrm{b}} \neq 0$ is then free to evolve in a 3D space).
}

based on Laskar's (1993) Frequency Map Analysis (FMA). The FMA method uses the diffusion rates of intrinsic frequencies as a measure for stability. The numerical values of these frequencies are provided by this method. The lowest intrinsic frequencies determine the necessary integration time. It is, therefore, possible that the FMA method requires longer integration times than MEGNO.

While the necessary integration time for FMA is provided by the lowest intrinsic frequency, one is a priori free to choose the time when applying MEGNO. The advantage is that a shorter integration time can be used. On the other hand, there is an uncertainty about the good choice of the integration time which might be too short. We, therefore, produce maps at different instant of times and consider the evolution of the most stable regions. Figures $1 \mathrm{a}$ and $\mathrm{b}$ show newly-obtained $\left[i_{\mathrm{r}}, \Omega_{\mathrm{r}}\right]$ maps for the HD 73526 system at 5000 and 50000 years respectively. While the first island (1) of Fig. 1a completely disappears in Fig. 1b, the second one remains always highly stable. Continuing in time, island (2) persists. We also find three very small stable islands (3) $-(5)$. One of the islands (3) is separated by $180^{\circ}$ in $\Omega_{\mathrm{r}}$ from the center of the large island (2). The two other islands, (4) and (5), are distributed symmetrically with respect to the islands (2) and (3). As a consequence, due to the lifetime and size of each stability zone, the large island (2) with $i_{\mathrm{r}} \in\left[173^{\circ}, 187^{\circ}\right]$ and $\Omega_{\mathrm{r}} \in\left[173^{\circ}, 266^{\circ}\right]$ contains the most stable orbits (i.e. the least "model dependent" on added perturbations) ${ }^{6}$. This does not necessarily imply that the two observational planets of the HD 73526 system are counter-revolving planets ${ }^{7}$. As a consequence, we study in the following section whether such a counter-revolving configuration is consistent with observational data.

\footnotetext{
${ }^{6}$ Let us note that a relative inclination around $180^{\circ}$ is equivalent to a planar problem where one planet has a retrograde motion with respect to the other. Therefore, considering a scale change of $180^{\circ}$ in relative inclinations, we will use the notation $i_{\mathrm{r}}^{\text {retro }}=1^{\circ}$ instead of $i_{\mathrm{r}}=179^{\circ}$, in the following.

7 Counter-revolving planets mean that the orbital elements of the two planets are orbiting in opposite directions about the central star.
} 


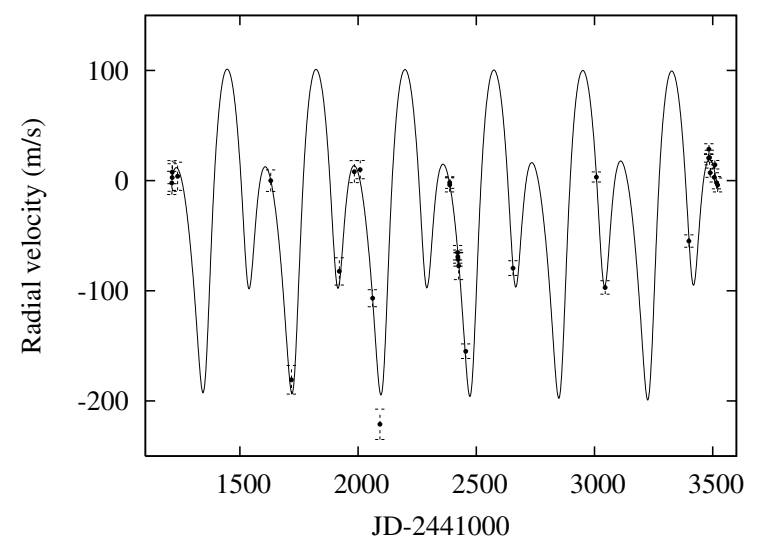

Fig. 2. Dynamical velocity fit with measured velocities of the HD 73526 planetary system. The best dynamical fit leading to a stable two-planet system is obtained for: $m_{\mathrm{b}}=2.4921 M_{\mathrm{Jup}}, m_{\mathrm{c}}=2.5919 M_{\mathrm{Jup}}, P_{\mathrm{b}}=$ 187.935 days, $P_{\mathrm{c}}=379.795$ days, $a_{\mathrm{b}}=0.6593 \mathrm{AU}, a_{\mathrm{c}}=1.0538 \mathrm{AU}$, $e_{\mathrm{b}}=0.2401, e_{\mathrm{c}}=0.2048, i_{\mathrm{b}}=0^{\circ}, i_{\mathrm{c}}=180^{\circ}, \Omega_{\mathrm{b}}=0^{\circ}, \Omega_{\mathrm{c}}=0^{\circ}$, $\omega_{\mathrm{b}}=184.569^{\circ}, \omega_{\mathrm{c}}=58.545^{\circ}, M_{\mathrm{b}}=97.297^{\circ}$, and $M_{\mathrm{c}}=221.361^{\circ}$. The velocity offset is: $V_{0}=-25.201 \mathrm{~m} \mathrm{~s}^{-1}$. The corresponding rms residuals are $6.34 \mathrm{~m} \mathrm{~s}^{-1}$ while the $\chi^{2}$ reduced factor is equal to 1.257 .

\section{Observational data fits}

Error-bars in published, orbital elements are significant and a fit including new observations may yield quite different orbital elements. By using different orbital elements that are consistent with observational data, Sándor et al. (2007) found stability for the coplanar and prograde case. On the one hand, for lowest values of $\chi^{2}$ and rms $\left(\chi^{2}=1.57\right.$ and $\mathrm{rms}=7.9 \mathrm{~m} \mathrm{~s}^{-1}$ for the best dynamical fit of Tinney et al. 2006), we find a weak chaotic solution. On the other hand, Sándor et al. (2007) obtain four stable solutions but with values of $\chi^{2}$ and $\mathrm{rms}$ somewhat higher than those of Tinney $\left(\chi^{2} \in[1.58 ; 1.87]\right.$ and $\left.\mathrm{rms} \in[8.04 ; 8.36]\right)$. As a consequence, the right astrometric characterization of the HD 73526 planetary system still remains open.

We have performed orbital fits for counter-revolving configurations using a genetic algorithm (called Pikaia; see Charbonneau 1995) based on a fitting method ${ }^{8}$. We find stable retrograde solutions for values of $\chi^{2}$ and rms smaller than the prograde fits of Tinney et al. (2006) and Sándor et al. (2007) $\left(\chi^{2}=1.257\right.$ and $\left.\mathrm{rms}=6.34 \mathrm{~m} \mathrm{~s}^{-1}\right)$. The radial velocity curve of the best stable fit is shown in Fig. 2. It is very similar to the radial velocity curve given by Tinney et al. (2006) and Sándor et al. (2007). As a consequence, we point out that the possibility of counter-revolving planets should not be discarded in observational-data fits.

Nevertheless, whatever the directions of motions of the two planets are, the $\chi^{2}$ values are significantly above the expected value of 1.0. More observations would enable better fits to be derived. However in these conditions, the possibility may not be excluded that the HD 73526 planetary system is a counterrevolving system. Anyway, the counter-revolving configuration related to the HD 73526 planetary system is consistent with the observational data. From a dynamical point of view, counterrevolving orbits are all the more plausible because they have larger highly stable regions.

\footnotetext{
${ }^{8}$ Orbital fitting process are notably explained in Beaugé et al. (2007).
}

Table 2. Statistical results about possibility of stable systems to be in retrograde resonance. 1000 random systems have been integrated in their errors bars and assuming prograde coplanar orbits or retrograde ones. The number of stable systems is indicated in each case. Data come from Tinney et al. (2006), Mayor et al. (2004), Vogt et al. (2005), McCarthy et al. (2004) and Correia et al. (2005; Table 4) respectively.

\begin{tabular}{cccc}
\hline \hline System sources & Period ratio & $\begin{array}{c}\text { Prograde } \\
\text { MMR }\end{array}$ & $\begin{array}{c}\text { Retrograde } \\
\text { MMR }\end{array}$ \\
\hline HD 73526 & $2 / 1$ & 17 & 500 \\
HD 82943 & $2 / 1$ & 755 & 1000 \\
HD 128311 & $2 / 1$ & 249 & 137 \\
HD 160691 & $5 / 1$ & 0 & 320 \\
HD 202206 & $5 / 1$ & 0 & 631 \\
\hline
\end{tabular}

\section{Statistical approach}

By integrating 1000 random systems (according to a MonteCarlo method) within the error-bars proposed by Tinney et al. (2006), we obtain statistically more stable solutions for coplanar counter-revolving orbits than for prograde ones. For prograde coplanar orbits, we find only 17 stable systems while for counterrevolving coplanar orbits, we obtain 500 stable systems.

The occurence of stable counter-revolving systems also appears in the neighborhood of other two-planet systems. The statistical results for their stability in the prograde case and in the counter-revolving one are presented in Table 2 for two additional 2:1 and two 5:1 resonance cases. In all cases, a significant number of stable systems in retrograde resonances is found. The high statistical occurence of stable retrograde configurations justifies the study of such solutions, whether or not they correspond at present to observational data. On the other hand, we are well aware of the cosmogonic problem for obtaining planets in retrograde resonances within the frame of current theories of planetary formation. During the early dynamical evolution of planetary systems, planets may end up on retrograde orbits (as discussed in the following section).

\section{Formation of counter-revolving planets}

Up to now, few works have been carried out on the formation of highly-inclined or counter-revolving orbits. For instance, Thommes \& Lissauer (2003) showed that a planetary migration leading to resonance capture in 2:1 MMR may cause a significant increase in the mutual inclinations of the planets. However, the resulting configuration never seems to exhibit retrograde motions. That is why, in this section, we propose two novel mechanisms of formation of counter-revolving configurations.

It has been known for a few years that free-floating planetary-mass objects have been located in interstellar space (see for instance Zapatero Osorio et al. 2000; and Lucas et al. 2005). These free-floating planets may interact with planetary systems in their host cluster; they are either scattered or captured. The recent studies of Varvoglis (2008) show that by integrating the trajectories of planet-sized bodies that encounter a coplanar, two-body system (a Sun-like star and a Jupiter mass), the probability of capture is significant, and almost half of the temporary captures are found to be of the counter-revolving type. Although captures of free-floating planets remain speculative, this could be a feasible mechanism for generating counter-revolving orbits.

Forming close-in planets by using the slingshot model revisited by Nagasawa et al. (2008) is another possibility. Starting from a hierarchical 3-planet system and considering a migration mechanism including process of planet-planet scattering 
(a)

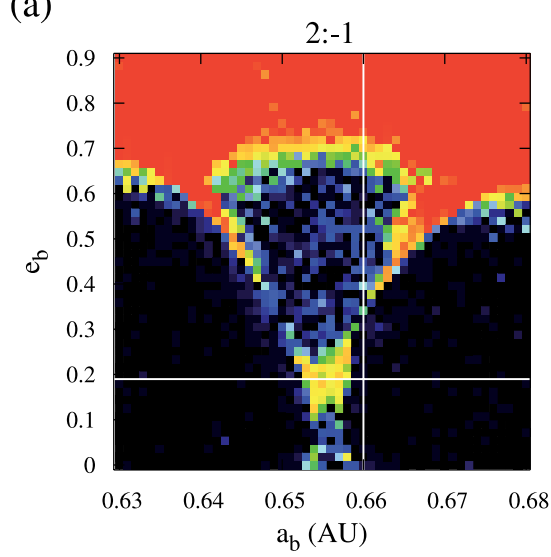

(b)

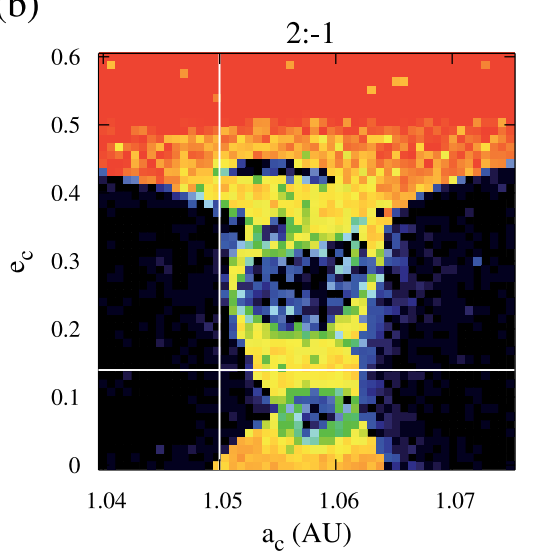

Fig. 3. Stability maps in the $\left[a_{\mathrm{b}}, e_{\mathrm{b}}\right]$ and $\left[a_{\mathrm{c}}, e_{\mathrm{c}}\right]$ parameter spaces based on the HD 73526 planetary system, taking into account initial conditions $(C)$ $\left(i_{\mathrm{r}}^{\text {retro }}=1^{\circ}\right.$ and $\left.\Omega_{\mathrm{r}}=216^{\circ}\right)$. Color scale is the same as in Fig. 1. Resolution of the grid is $50 \times 50$. The pseudo-observational point is indicated by the intersection of horizontal and vertical lines. $V$-shape structures correspond to the 2:1 retrograde MMR (2:-1 MMR).

and tidal circularization, the authors show indeed that close-in planets may be formed. In a few cases, due to the Kozai mechanism (involving exchanges between eccentricities and inclinations), one planet enters a retrograde motion.

Considering (1) these two feasible mechanisms of formation of counter-revolving orbits, (2) the dynamical fit obtained in Sect. 3, and (3) the statistical occurence of retrograde solutions (Sect. 4), we may say that counter-revolving theoretical configurations are serious candidates for real systems (that could be observed later). In the future, we will study these two formation processes more deeply. In the following sections, we focus on the specific dynamical behavior of systems harboring counterrevolving planets.

\section{Fine structure of the resonance}

Due to the retrograde motion of planet $c^{9}$, the orbital resonance of a given planetary system (e.g. the HD 73526 planetary system) is called a 2:1 retrograde MMR (that we also annotate as a 2:-1 MMR). Studying the fine structure of this MMR provides a key to understand the stability of the system. We assume initial conditions taken from the stability island (2) of the $\left[i_{\mathrm{r}}, \Omega_{\mathrm{r}}\right]$ map (Fig. 1b), that is to say the elements of Table 1 and in addition $\Omega_{\mathrm{r}}=216^{\circ}$ and $i_{\mathrm{r}}^{\mathrm{retro}}=1^{\circ}$. This set of initial conditions is noted $(\mathrm{C})^{10}$. We then obtain the two $\left[a_{\mathrm{b}}, e_{\mathrm{b}}\right]$ and $\left[a_{\mathrm{c}}, e_{\mathrm{c}}\right]$ stability maps presented in Fig. 3. The presence of a strong MMR generates clear instability zones with a prominent $V$-shape structure in Fig. 3a. We note the narrowness of the $V$-shapes, approximately $0.006 \mathrm{AU}$ wide for the inner orbit (when $e_{\mathrm{b}}=0$ ) and $0.0015 \mathrm{AU}$ wide for the outer one (when $e_{\mathrm{c}}=0$ ). As a comparison, $V$-shape structures of the Sun-Jupiter-Saturn system are five times and twice as large respectively (for Jupiter and Saturn). We note in addition how the pseudo-observational point lies at the edge of the $V$-shapes (Fig. 3).

\footnotetext{
9 By convention, the orbital plane of the planet $b$ is chosen as a reference plane. The planet $\mathrm{b}$ is supposed moving in the prograde direction while the planet $\mathrm{c}$ in the retrograde direction. Results remain analoguous with the reverse assumption (b retrograde and c prograde).

${ }^{10}$ For $\Omega_{\mathrm{r}}=216^{\circ}$ and $i_{\mathrm{r}}^{\text {retro }}=1^{\circ}$, the value of $\chi^{2}$ obtained with the velocity offset $V_{0}=-38 \mathrm{~m} \mathrm{~s}^{-1}$ is 2.44 . The rms residuals are 12.31 .
}

\section{Evidence for a retrograde resonance}

Maps for extrasolar-planet systems, with stability regulated by a prograde MMR, are characterized by small islands of stability (or linear stable strips in $\left[a_{\mathrm{b}}, a_{\mathrm{c}}\right]$ maps) inside large zones of instability. By contrast, in the case of retrograde resonance and when assuming the initial conditions $(\mathrm{C})$, we detect a dense, stable regime in a series of maps (e.g. $\left.\left[a_{\mathrm{b}}, a_{\mathrm{c}}\right]\right)$, except for one unstable zone related to the MMR. In several cases of resonant prograde systems, it has been shown that planets on highly stable orbits may avoid close approaches due to their adequate positions over their orbits and apsidal line locking (see Bois et al. 2003). This mechanism of stability is not lost during the dynamical evolution of the system when the apsidal lines on average precess at the same rate (i.e. the ASP phenomenon). Without such a protection mechanism of 2:1 MMR combined to an ASP, disturbing close approaches between the planets are theoretically possible in various planet positions. In the case of the $2: 1$ retrograde MMR, one planet being retrograde, orbital motions occur then in opposite directions. As a consequence, the length of time that planets spend in conjunction, is much shorter for counterrevolving orbits than for prograde ones. This could explain the narrowness of the $V$-shapes in Fig. 3.

In Gayon \& Bois (2008), we showed that, in cases of very compact planetary systems obtained by a scale reduction of a given observed system, "retrograde" stable islands survive, in contrast to "prograde" ones that disappear. This scale reduction and the $V$-shapes of Fig. 3 illustrate the efficiency of retrograde MMRs for providing stability. In addition, this MMR mechanism is coupled to specific behaviors of the apsidal lines, as shown in the following section.

\section{A new mechanism of apsidal precession at retrograde resonance}

In the case of the 2:1 retrograde MMR (2:-1 MMR), the expressions for the resonance angles $\theta_{1}$ and $\theta_{2}$, and the ASP angle $\theta_{3}$ are:

$\theta_{1}=-\lambda_{\mathrm{b}}-2 \lambda_{\mathrm{c}}+3 \tilde{\omega}_{\mathrm{b}}$
$\theta_{2}=-\lambda_{\mathrm{b}}-2 \lambda_{\mathrm{c}}+3 \tilde{\omega}_{\mathrm{c}}$
$\theta_{3}=3\left(\tilde{\omega}_{\mathrm{b}}-\tilde{\omega}_{\mathrm{c}}\right)$

where $\lambda$ is the mean longitude, and $\tilde{\omega}$ is the apsidal longitude, defined by $\lambda=M+\tilde{\omega}$ and $\tilde{\omega}=\Omega+\omega$ respectively, for prograde 


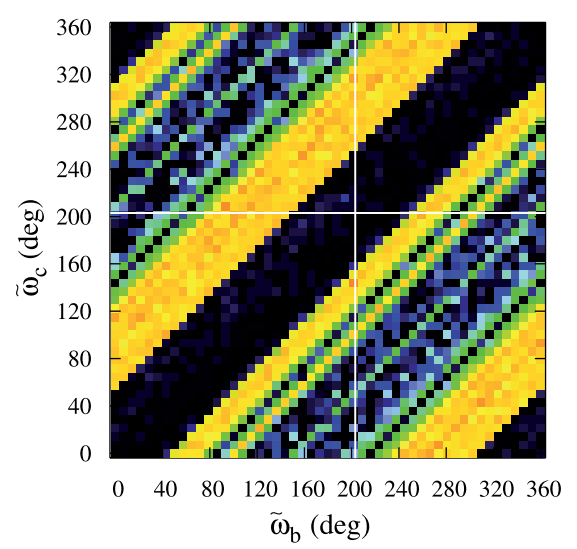

Fig. 4. Stability map in the $\left[\tilde{\omega}_{\mathrm{b}}, \tilde{\omega}_{\mathrm{c}}\right]$ parameter space based on the HD 73526 planetary system taking into account initial conditions $(C)$ $\left(i_{\mathrm{r}}^{\text {retro }}=1^{\circ}\right.$ and $\left.\Omega_{\mathrm{r}}=216^{\circ}\right)$. The pseudo-observational point is inside the stability linear strip. Color scale is the same as in Fig. 1. Resolution of the grid is $50 \times 50$.

orbital motion of a planet; for retrograde motion, these variables are defined to be: $\lambda=-M+\tilde{\omega}$ and $\tilde{\omega}=\Omega-\omega$. The general expression for the relative apsidal longitude is: $\Delta \tilde{\omega}=\tilde{\omega}_{\mathrm{b}}-\tilde{\omega}_{\mathrm{c}}=\theta_{3} / q$, where $q$ is the order of the resonance. A more thorough investigation of retrograde resonances, using an analytical approach, is in preparation (Gayon et al. 2008).

In Fig. 4 we plot the $\left[\tilde{\omega}_{\mathrm{b}}, \tilde{\omega}_{\mathrm{c}}\right]$ parameter space that shows a stable linear strip, in dark-blue, including the "pseudoobservational" point. We learn that stable solutions are possible only when $\tilde{\omega}_{\mathrm{b}}$ and $\tilde{\omega}_{\mathrm{c}}$ precess, on average, at the same rate. The stabilizing mechanism of the system involves a synchronous precession of the apsidal lines. The two longitudes of periastron do not precess however in the same direction. The outer orbit is affected by a retrograde precession $\left(-\tilde{\omega}_{\mathrm{c}}\right)$ relative to the inner orbit's precession $\left(\tilde{\omega}_{\mathrm{b}}\right)$ (see Figs. 5c vs. b). Writing the longitudes of periastron as directed angles, we find that the relative apsidal longitude $\Delta \tilde{\omega}$ neither circulates nor librates clearly, as shown in Fig. 5a. $\Delta \tilde{\omega}$ presents a strange motion composed of: (1) a phase of prograde circulation including librations with amplitudes of $\pm 8^{\circ}$, then following a sharp reversal of circulation direction, (2) a phase of fast retrograde circulation, until a second, sharp reversal. These two phases alternate successively according to alternations (or rocking) of $180^{\circ}$, which correspond to a sort of $\operatorname{cusp}^{11}$. We note that $\tilde{\omega}_{\mathrm{c}}$, in contrast to the case for $\tilde{\omega}_{\mathrm{b}}$, does not uniformly circulate but presents retrograde circulation phases interrupted with short libration intervals (Figs. 5c vs. b). In spite of the opposite directions of their precession, both orbits precess, on average, at the same rate. As a consequence, the planetary system is affected by an apsidal synchronous precession. Considering the alternating behavior of the $\Delta \tilde{\omega}$ angle or the unusual presence of cusp in the $\Delta \tilde{\omega}$ behavior, we refers to this new stabilizing factor as either an alternating ASP, or a rocking ASP (or RASP). We have produced movies illustrating the mechanisms introduced in the present paper, in particular the phenomenon of RASP ${ }^{12}$.

\footnotetext{
11 At phase transitions, a scattering of dots appears. When the outerorbit eccentricity goes to zero, the $\tilde{\omega}_{\mathrm{c}}$ angle that depends on the $(a-r) / a e$ ratio is not defined.

12 Movies are downloadable from: http://www.oca.eu/gayon/ Extrasolar/Retro_MMR/movies.html and

http://www. aanda.org
}
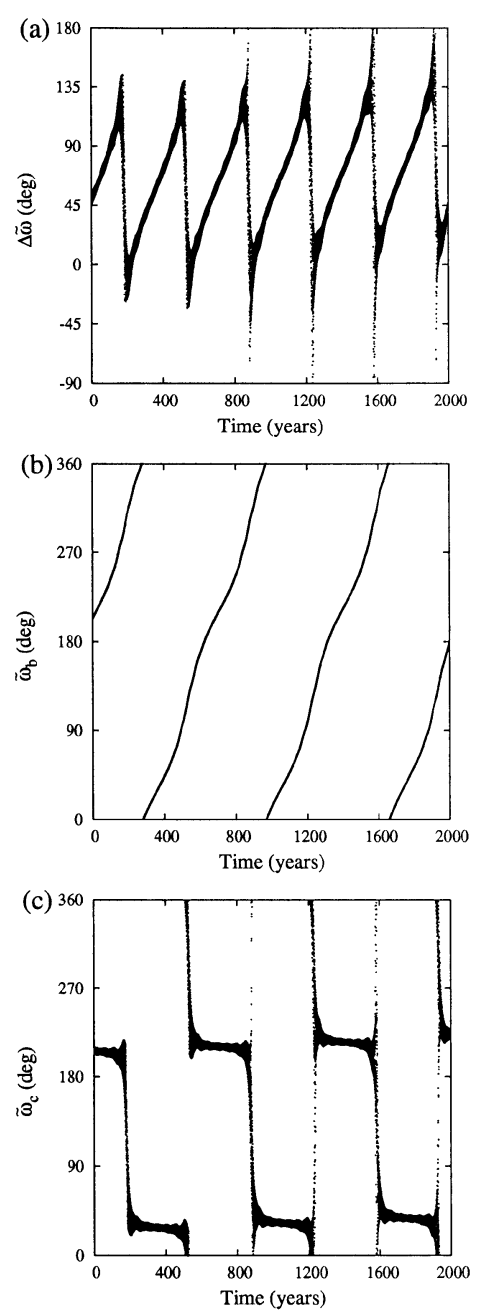

Fig. 5. Time variation of the $\Delta \tilde{\omega}$ angle a), $\tilde{\omega}_{\mathrm{b}} \mathbf{b}$ ), and $\tilde{\omega}_{\mathrm{c}} \mathbf{c}$ ) for initial conditions $(C)$. In panel $\mathbf{b}$ ), the slope of $\tilde{\omega}_{\mathrm{b}}$ is positive while the one of $\tilde{\omega}_{\mathrm{c}}($ panel c) $)$ is negative. Panel a) expresses the behavior of the $\Delta \tilde{\omega}$ combination of $\tilde{\omega}_{\mathrm{b}}$ and $\tilde{\omega}_{\mathrm{c}}$ (see text).

Relations between the eccentricity of the inner orbit and the $\Delta \tilde{\omega}$ angle exist. In $\left[\Delta \tilde{\omega}, e_{\mathrm{b}}\right]$ parameter space, we can see from Fig. 6 how the 2:1 retrograde MMR spreads out its resources:

(1) Inside the 2:-1 MMR (i.e. inside the $[a, e] V$-shape), both apsidal lines on average precess at the same rate and in the same prograde direction. The 2:1 retrograde MMR is then combined with a uniformly prograde ASP (island (1)).

(2) Close to the 2:-1 MMR (i.e. outside but close to the $[a, e]$ $V$-shape), both apsidal lines on average precess at the same rate but in opposite directions. The 2:1 retrograde nearMMR is combined with the mechanism of rocking ASP (island (2)).

(3) The division between these two islands is related to the degree of closeness to the 2:1 retrograde MMR. We highlight the fact that, for a long timescale, the third island in the $\left[\Delta \tilde{\omega}, e_{\mathrm{b}}\right]$ map, is proved to be a chaotic zone (island (3) where $\left.\Delta \tilde{\omega} \in\left[80^{\circ}, 280^{\circ}\right]\right)$.

We note that a mechanism of stability involving an ASP may persist far from the MMR in the prograde case, while it disappears for short distance to the MMR in the counter-revolving case. Hence, moving away from the $[a, e] V$-shape of the 2:-1 MMR 


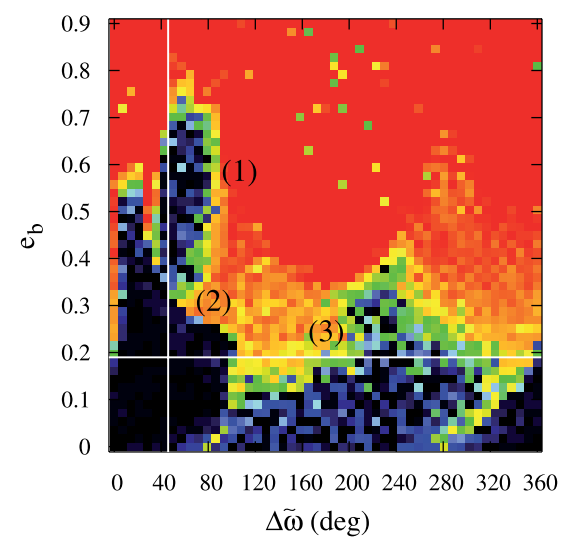

Fig. 6. Stability map in the $\left[\Delta \tilde{\omega}, e_{\mathrm{b}}\right]$ parameter space based on the HD 73526 planetary system, taking into account initial conditions $(C)$ (Table 1 with in addition $i_{\mathrm{r}}^{\text {retro }}=1^{\circ}$ ). The pseudo-observational point is inside the stability large island (2) characterized by a 2:1 retrograde near-resonance and an alternating ASP. Color scale is the same as in Fig. 1. Resolution of the grid is $50 \times 50$.

(Fig. 3), we find that both apsidal lines precess in opposite directions but at different rates.

By studying the parameter-space in the vicinity of the best-fit of the HD 73526 planetary-system and searching for stable configurations with similar values of rms, a new, theoretical mechanism of stability has been discovered. It is characterized by a 2:1 retrograde, near-MMR combined to a rocking ASP. Such a stability also allows a large range of eccentricities (see for instance planet b in Fig. 6). Such a mechanism is particularly robust on large timescales. It is why such mechanisms involving such resources of the 2:-1 orbital resonance could prove to be relatively generic and suitable for the stability of a class of compact multi-planetary systems where other solutions of the 3-body problem are not possible.

\section{The 5:1 retrograde MMR}

The mechanism of stability involving both a retrograde MMR and an ASP is also found for the 5:1 orbital period ratio by scanning initial conditions in the vicinity of the HD 160691 planetary system (McCarthy et al. 2004, planets b and c, see Table 1). By detecting a fourth planet, we note that new observations of this system have modified the orbital structure of the entire system (Pepe et al. 2007). The observations of Pepe et al. (2007) show furthermore that a new coplanar fit of prograde orbits and a new fourth planet seems to solve the problem without the need for retrograde resonance. We note that in the vicinity of the best fit solution of McCarthy et al. (2004), it is possible to find an example of 5:1 retrograde resonance. We consider this possibility as an academic investigation of this order of MMR.

The important point is that this 3-body system is completely unstable for prograde orbits. Nevertheless, by scanning the nondetermined parameter space, only one island of stability exists (for $i_{\mathrm{r}} \in\left[156^{\circ}, 204^{\circ}\right]$ and $\Omega_{\mathrm{r}} \in\left[7^{\circ}, 180^{\circ}\right]$ ) and after an analysis we highlight the retrograde motion of the outer planet. Moreover, scanning the $\left[\Delta \tilde{\omega}, e_{\mathrm{b}}\right]$ parameter space of the system in 5:1 retrograde MMR, as for the HD 73526 planetary system in 2:1 retrograde MMR, we observe the distribution of the different apsidal behaviors (see Fig. 7). Islands (1) and (3) are characterized by an ASP with an apsidal alignement. More precisely, both longitudes of periastron $\left(\tilde{\omega}_{\mathrm{b}}\right.$ and $\left.\tilde{\omega}_{\mathrm{c}}\right)$ on average precess at the same rate, both in the retrograde direction, while the

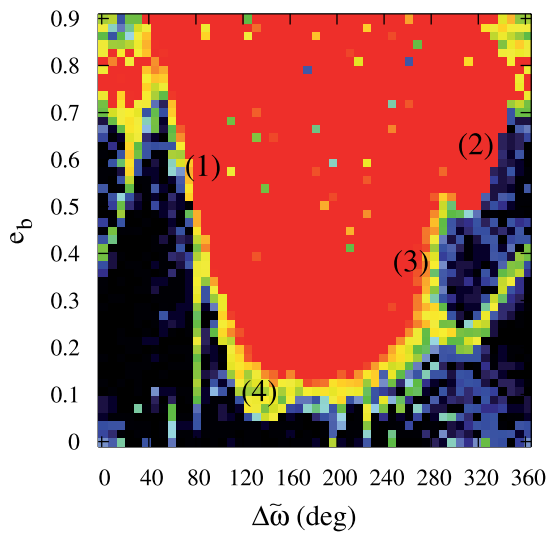

Fig. 7. Stability map in the $\left[\Delta \tilde{\omega}, e_{\mathrm{b}}\right]$ parameter space based on the HD 160691 planetary system (data from McCarthy et al. (2004) shifted to the 5:1 retrograde MMR (i.e. $a_{\mathrm{b}}=1.44 \mathrm{AU}$ ), see Table 1 with in addition $i_{\mathrm{r}}^{\text {retro }}=1^{\circ}$ ). Islands (1) and (3) are characterized by a 5:-1 MMR and an ASP with apsidal alignement while islands (2) and (4) by an 5:-1 MMR and a circulation of the $\Delta \tilde{\omega}$ variable. Color scale is the same as in Fig. 1. Resolution of the grid is $50 \times 50$.

$\Delta \tilde{\omega}$ variable librates about $0^{\circ}:$ it is a uniformly retrograde ASP. Within islands (2) and (4), $\tilde{\omega}_{\mathrm{b}}$ and $\tilde{\omega}_{\mathrm{c}}$ precess in opposite directions but according to different rates: $\Delta \tilde{\omega}$ circulates. Hence, no apsidal line locking is required for stability. We find fine, $V$-shape structures in $[a, e]$ maps corresponding to the 5:1 retrograde MMR. The width is $0.02 \mathrm{AU}$ for the inner planet (when $e_{\mathrm{b}}=0$ ) and only $0.002 \mathrm{AU}$ for the outer one (when $e_{\mathrm{c}}=0$ ). Consequently, the dynamical study of this case derived from the HD 160691 planetary system allows us to find another theoretical possibility of stability involving resources of a retrograde MMR.

\section{Conclusion}

We have found novel mechanisms giving rise to stability that could be suitable for a class of compact planetary systems. Such mechanisms involve counter-revolving orbits forming a retrograde MMR occuring in a quasi-identical plane. High statistical occurence of stable counter-revolving orbits is found. Our study of retrograde MMRs indicates the large stability domains and the specific behaviors of the precession and resonant angles. We propose that these large stability domains are caused by close approaches much faster and shorter for counter-revolving configurations than for the prograde ones. Scanning the HD 73526 planetary system, we find evidence for a new type of apsidal precession (the rocking ASP). We find that the difference between the longitudes of periastron reveals a specific alternation mode at retrograde resonances. We emphasize that the counterrevolving configuration studied for the HD 73526 planetary system is consistent with the observational data. Free-floating planets or the Slingshot model might explain the origin of such counter-revolving systems.

Acknowledgements. We thank Alessandro Morbidelli, Makiko Nagasawa, Hans Scholl, and Charalampos Varvoglis for useful discussions. We particularly thank Cristián Beaugé for providing us a code performing orbital fits and, as a referee, for very constructive comments that greatly helped to improve the paper.

\section{References}

Beaugé, C., Ferraz-Mello, S., \& Michtchenko, T. A. 2003, ApJ, 593, 1124 Beaugé, C., Michtchenko, T. A., \& Ferraz-Mello, S. 2006, MNRAS, 365, 1160 
Beaugé, C., Ferraz-Mello, S., \& Michtchenko, T. A. 2007, in Exoplanets, ed. R. Dvorak (Wiley-VCH), 1

Bois, E. 2005, ESA Science Requierements Document, to be appeared

Bois, E., Kiseleva-Eggleton, L., Rambaux, N., \& Pilat-Lohinger, E. 2003, ApJ, 598, 1312

Bois, E., Rambaux, N., Kiseleva-Eggleton, L., \& Pilat-Lohinger, E. 2004, ASP Conf. Proc. 321, ed. J. P. Beaulieu, A. Lecavelier des Etangs, \& C. Terquem, 349

Bois, E., Rambaux, N., Kiseleva-Eggleton, L., \& Pilat-Lohinger, E. 2005, 1st ISSI Team Meeting, in Evolution of habitable planets, ed. H. Lammer, in press

Charbonneau, P. 1995, ApJS, 101, 309

Chiang, E. I., \& Murray, N. 2002, ApJ, 576, 473

Cincotta, P., \& Simó, C. 2000, A\&AS, 147, 205

Cincotta, P., \& Giordano, C. 2000, Advances in Space Dynamics 2: Applications in Astronomy, ed. O. C. Winter, \& A. F. B. A. Prado (São José dos Campos: Inst. Nac. Pesquisas Espaciais), 237

Cincotta, P., Giordano, C., \& Simó, C. 2002, Physica D, 182, 151

Correia, A. C. M., Udry, S., Mayor, M., et al. 2005, A\&A, 440, 751

Ferraz-Mello, S., Michtchenko, T. A., Beaugé, C., \& Callegari, N. 2005a, Lecture Notes Phys., 683, 219

Ferraz-Mello, S., Michtchenko, T. A., \& Beaugé, C. 2005b, ApJ, 621, 473

Fischer, D. A., Marcy, G. W., Butler, R. P., Laughlin, G., \& Vogt, S. S. 2002, ApJ, 564, 1028

Fischer, D. A., Marcy, G. W., Butler, R. P., et al. 2003, ApJ, 586, 1394

Gayon, J., \& Bois, E. 2008, Exoplanets: detection, formation and dynamics: Proceeding of the 249th Symposium of the International Astronomical Union, Cambridge University Press, ed. Y. S. Sun, S. Ferraz-Mello, \& J. L. Zhou, to be appeared

Gayon, J., Bois, E., \& Scholl, H. 2008, Cel. Mech. Dyn. Astron., Special Issue: Theory and Applications of Dynamical Systems, submitted
Hadjidemetriou, J. D. 2002, Cel. Mech. Dyn. Astron., 83, 141

Ji, J., Kinoshita, H., Liu, L., Li, G., \& Nakai, H. 2003, Cel. Mech. Dyn. Astron., 87,113

Ji, J., Kinoshita, H., Liu, L., \& Li, G. 2007, ApJ, 657, 1092

Kiseleva-Eggleton, L., Bois, E., Rambaux, N., \& Dvorak, R. 2002, ApJ, 578, L145

Laskar, J. 1993, Physica D, 67, 257

Lee, M. H., \& Peale, S. J. 2002, ApJ, 567, 596

Lee, M. H., \& Peale, S. J. 2003, ApJ, 592, 1201

Libert, A. S., \& Henrard J. 2006, Icarus, 183, 186

Lucas, P. W., Roche, P. F., \& Tamura, M. 2005, MNRAS, 361, 211

Marzari, F., Scholl, H., \& Tricarico, P. 2006, A\&A, 453, 341

Mayor, M., Udry, S., Naef, D., et al. 2004, A\&A, 415, 391

McArthur, B. E., Endl, M., Cochran, W. D., et al. 2004, ApJ, 614, L81

McCarthy, C., Butler, R. P., Tinney, et al. 2004, ApJ, 617, 575

Michtchenko, T. A., Ferraz-Mello, S., \& Beaugé, C. 2006, Icarus, 181, 555

Nagasawa, M., Ida., S., \& Bessho, T. 2008, ApJ, accepted [arXiv:0801.1368v1]

Pepe, F., Correia, A. C. M., Mayor, M., et al. 2007, A\&A, 462, 769

Psychoyos, D., \& Hadjidemetriou, J. D. 2005, Cel. Mech. Dyn. Astron., 92, 135

Rivera, E. J., Lissauer, J. J., Butler, R. P., et al. 2005, BAAS, 37, 1487

Sándor, Z., Kley, W., \& Klagyivik, P. 2007, A\&A, 472, 981

Thommes, E. W., \& Lissauer, J. J. 2003, ApJ, 597, 566

Tinney, C. G., Butler, R. P., Marcy, G. W., et al. 2006, ApJ, 647, 594

Varvoglis, C. 2008, Exoplanets: detection, formation and dynamics: Proceeding of the 249th Symposium of the International Astronomical Union (Cambridge University Press), ed. Y. S. Sun, S. Ferraz-Mello, \& J. L. Zhou, to be appeared Vogt, S. S., Butler, R. P., Marcy, G. W., et al. 2005, ApJ, 632, 638

Zapatero Osorio, M. R., Béjar, V. J. S., Martín, E. L., et al. 2000, Science, 290, 103 